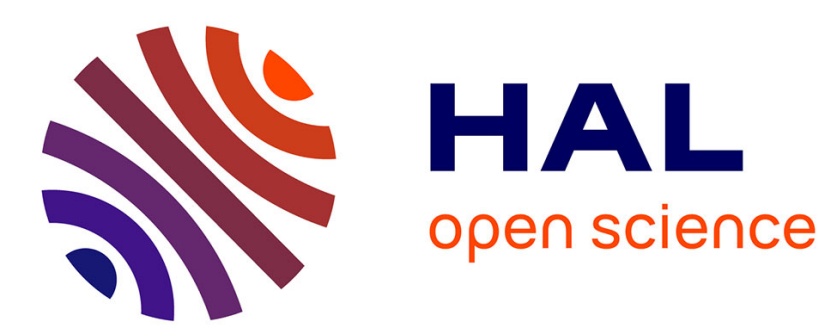

\title{
Identification of a PEMFC fractional order model
}

Miassa Amira Taleb, Olivier Béthoux, Emmanuel Godoy

\section{To cite this version:}

Miassa Amira Taleb, Olivier Béthoux, Emmanuel Godoy. Identification of a PEMFC fractional order model. International Journal of Hydrogen Energy, 2016, 42 (2), pp.1499-1509. 10.1016/j.ijhydene.2016.07.056 . hal-01385072

\section{HAL Id: hal-01385072 \\ https://hal.science/hal-01385072}

Submitted on 24 Oct 2016

HAL is a multi-disciplinary open access archive for the deposit and dissemination of scientific research documents, whether they are published or not. The documents may come from teaching and research institutions in France or abroad, or from public or private research centers.
L'archive ouverte pluridisciplinaire HAL, est destinée au dépôt et à la diffusion de documents scientifiques de niveau recherche, publiés ou non, émanant des établissements d'enseignement et de recherche français ou étrangers, des laboratoires publics ou privés. 


\section{Accepted Manuscript}

Identification of a PEMFC fractional order model

Miassa Amira Taleb, Olivier Béthoux, Emmanuel Godoy

DOI:

10.1016/j.ijhydene.2016.07.056

Reference:

Publisher:

ELSEVIER

To appear in:

\section{International Journal of HYDROGEN ENERGY}

Received date: $\quad 21$ February 2016

Revised date: $\quad 10$ June 2016

Accepted date: $\quad 11$ July 2016

Date of Publication: $\quad 28$ September 2016 (available on line)

Please cite this article as: Miassa Amira Taleb, Olivier Béthoux, Emmanuel Godoy, Identification of a PEMFC fractional order model, International Journal of Hydrogen Energy, Available online 28 September 2016, ISSN 0360-3199, http://dx.doi.org/10.1016/j.ijhydene.2016.07.056.

(http://www.sciencedirect.com/science/article/pii/S0360319916306954) doi: 10.1016/j.ijhydene.2016.07.056

Document Version: Early version, also known as pre-print

This is a PDF file of an unedited manuscript that has been accepted for publication. As a service to our customers we are providing this early version of the manuscript. The manuscript will undergo copyediting, typesetting, and review of the resulting proof before it is published in its final form. Please note that during the production process errors may be discovered which could affect the content, and all legal disclaimers that apply to the journal pertain. 


\title{
Identification of a PEMFC fractional order model
}

\author{
Miassa Amira Taleb ${ }^{1}$, Olivier Béthoux ${ }^{1}$, Emmanuel Godoy $^{1}$ \\ Laboratoire des Signaux et Systmes (L2S, UMR CNRS 8506), CNRS - CentraleSupelec - Universit Paris-Sud, 3, rue Joliot Curie, 91192, \\ Gif-sur-Yvette, France \\ GeePs, 11, rue Joliot Curie,Plateau de Moulon, 91192 Gif sur Yvette Cedex \\ Miassa Amira Taleb ${ }^{a, b, *}$, Olivier Béthoux ${ }^{b}$, Emmanuel Godoy ${ }^{a}$ \\ ${ }^{a}$ Laboratoire des Signaux et Systmes (L2S, UMR CNRS 8506),CNRS - CentraleSupelec - Universit Paris-Sud, 3, rue Joliot Curie, 91192, \\ Gif-sur-Yvette, France \\ ${ }^{b}$ GeePs, 11, rue Joliot Curie,Plateau de Moulon, 91192 Gif sur Yvette Cedex
}

\begin{abstract}
The present paper addresses the important issue of monitoring the operating state of the Polymer Electrolyte Membrane Fuel Cell systems. The monitoring system takes a model based approach. Its originality lies in adopting a fuel cell fractional order impedance model which permits to provide a better insight into the fuel cell physical phenomena without increasing the number of parameters. This article first validates experimentally the accuracy of the suggested model, using a frequency identification method carried out by nonlinear optimization using single fuel cell experimental impedance spectroscopy data. In a second phase, time series identification is achieved using a least square method specifically designed for fractional order models. The latter method is first verified on registered data which represents a basic tool for off-line monitoring. Subsequently it is refined as a recursive tool permitting an on-line monitoring; it is validated on laboratory test bench.
\end{abstract}

Keywords:

Polymer Electrolyte Membrane Fuel cell, PEMFC fractional order model, FC parameter identification, diagnosis 2010 MSC: 00-01, 99-00

\section{Introduction}

Electric vehicle (EV) is an emerging and growing market projects gradually emerge and fuel cell (FC) technol- ${ }^{20}$ ogy may boost the EV industry providing "zero emission" 5 long-range vehicles and fast recharging ability. Indeed, in comparison with batteries, power and energy are independent:independent: the vehicle range is linked to the hydrogen reservoir which takes few minutes to fill while the ${ }^{25}$ onboard available power is dependent on the FC size. Con10 sequently FC represents an attractive opportunity for the future development of EV since hydrogen allows a much larger cruising range than battery and convenient recharge. In this respect Polymer Electrolyte Membrane (PEM) FC ${ }^{30}$ systems operate at low temperature and high power den15 sity and are ideal candidates for this application, which results incaracterizedcharacterized by heavy environmental

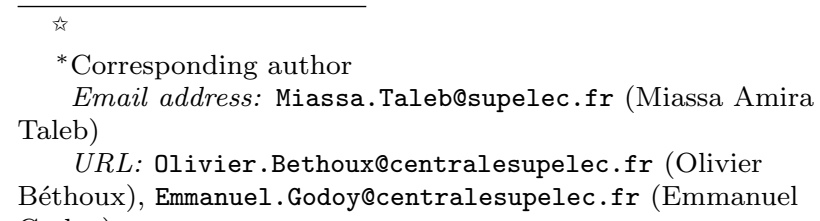

constraints with intermittent and highly fluctuating operation stop/start conditions They represent an attractive opportunity for the future development of electrical vehicles since hydrogen allows a much larger cruising range than battery and convenient recharge. Nevertheless, much remains to be done to overcome some of PEMFC technological obstacles which usually appear for automotive applications 11. Water management (to avoid Fflooding and dehydrationrying) $2,3,3,4,[5,6], 7,8]$ is one of the most critical points to solve enhance. The principal means by which this will be achieved are both design optimization [3], 6], 9] and health monitoring diagnosis and prognostics [10], 11], 12, [13, 7] in order for the control system to maintain normal FC operation. This is where the fuel cells PEMFC state operating real-time monitoring intervenes in order to establish a system diagnosis . Diagnosis methods [2, 12, [13, 14, can be applied in real time, coupled with a FC control system (On Board diagnosis) or during regular planned maintenance.

Diagnosis proposed approaches may include signal processing techniques, experiential learning methods and model based procedures [xx on peut mme se citer!]. The latter has the advantage of providing a tool easier to adapt 40 from one PEMFC design to another. But the main issues 
are to provide a reliable model [15] and to develop specific parameters identification tools. The aim of this paper is to propose accurate but compact model and robust iden-100 tification tools which can be used for real time system 45 diagnosis.

The aim of this paper is to propose a more compact and accurate model and robust identification tools which can be used for real time system diagnosis

This method uses, as far as possible, the least number 50 of sensors, by limiting the PEMFC state monitoring to the use of available data of the system such as the current and voltage. To this end, it is relevant to employ electric model built using the physical equations governing the $\mathrm{FC}_{110}$ process. Fontes [16, [17] proposed a FC large signal non-

55 linear model represented by an electrical circuit using voltage controlled current sources, reflecting the causality of phenomena. This model is commonly linearized to deduce an electrical impedance model. For improving Membrane ${ }_{115}$ Electrode Assembly (MEA) water management, N. Fou-

60 quet 11] diagnoses the FC operating state based on the sensitivity of certain parameters to the MEA drying or flooding. He uses an equivalent electrical circuit model based on the Randles model. He also changes the double ${ }_{120}$ layer capacitor with Constant Phase Element (CPE). Al${ }_{65}$ ternatively I. Sadli [18, 19, 20, models the FC behavior by an impedance, substituting an equivalent transmission line with $\mathrm{RC}$ distributed cells for the classic Randles circuit. In this way he can take into account the critical $\mathrm{AME}_{125}$ convection-diffusion phenomenon in the electrochemical

70 impedance. To interpret accurately it he needs to use a large number of individual RC cells. But partial differential equations correctly describe the convection-diffusion phenomenon. These latter leads to fractional order de-130 rives. That is the reason why Cao et al 21] proposes a

${ }_{75}$ fractional order model of solid oxide FC. The current work is also based on a model such as this. The key idea is to reformulate it so as to obtain a compact model characterized by a limited number of parameters. Experimental results ${ }_{135}$ conducted on a laboratory test bench will suggest that the

80 proposed model reproduces reality very well. Furthermore a detailed parameter analysis will permit to reduce the number of parameters to follow.

The analysis of the FC impedance is an interesting solution for identifying failures through their signatures de-

${ }_{85}$ formation of impedance spectrum [11, [22], [? ]. One of the most used methods for electrochemical system char-140 acterization and fuel cell diagnosis is the impedance spectroscopy [23, [11, 9], 22.

For instance, Kurz et al. 24] distinguish between flood90 ing and drying modes using the analysis of two specific frequencies, one low and one high. But, since based on a har-145 monic perturbation, frequency domain analysis remains time consuming especially in the case of FC where the minimum frequency value is in the range of $100 \mathrm{mHz}$. That

95 is the reason why it is crucial to develop alternative AC impedance technique identification. This article intends to150 create a time domain rapid method able to monitor in real time the FC model parameters as a primary tool for diagnosing purpose and control modifications. For this purpose, the parameters are identified by least square method adapted to the explicit fractional order FC model. In order to enable on-line investigations, the latter is adapted in a recursive form. Both time-series identification techniques represent basic tools for off-line and online diagnosis. They are experimented using a Pseudo Random Binary Sequence (PRBS) small current perturbation and measuring the FC voltage response which can be technically possible on a fuel cell working in a car.

Identification results of the different methods are presented, analyzed and discussed. These methods estimate the parameters describing the impedance spectrum evolution reflecting the fuel cell internal state. They represent basic tools for off-line and online diagnosis. Fractional order model identification results using these methods will judge the accuracy of the model to describe the fuel cell behavior. The structure of the present In this paper, is as follows. After this brief introduction setting the background and the context of the work, Section II discusses a fuel cell FC impedance model and section III reformulates the non-linear model to obtain an explicit fractional order transfer function. An analysis of the parameters sensitivity to flooding and drying will also be presented. Section IIIV presents the experimental setup used in the following section Part IV to identify the PEMFC model. In section IV an identification method based on frequency measurements is presented to validate the model. The results obtained are compared with spectrum measurements obtained experimentally, concluding in the sufficient precision and accuracy about the accuracy of the presented model. Section VI presents a second identification method of the fractional order model using time-series data. This method is derived from the classical least square and recursive least square methods and reformulated to be applied to the case of fractional order systems. Experimental results will be presented. Finally, the last section presents some conclusions and perspectives.

\section{2. introduction}

\section{PEMFC fractional order model}

\subsection{PEMFC impedance model}

Over the years, many FC models have been developed both to better understand the phenomena inside a FC system and to predict them 25], 26]. Our goal is to determine a PEMFC model allowing to implement a real-time FC monitoring with to the aim of PEMFC reversible failures diagnosis, such as water flooding or drying. In this context, a trade-off between accuracy and time execution has to be found. In the scientific literature, many studies use classical electrical impedance models to represent the FC system. For instance, Fontes [16], 17] studies interactions between FC and power converters based on an electrical impedance model. Similarly Fouquet [1] and 
Phlippoteau [22] deal with the diagnosis of FC by using two different electrical impedance models, on dedicated to small magnitude signal and the other to large magnitude signal 27. In 28 and 29 Hinaje and al. use electrical analogy to describe mass and charge transport in FC and build a model well adapted to describe FC interacting with its electric load. Finally, Sadli [19, [19, 20] refines the PEMFC impedance model as a as a constants distributed transmission line so as to take into account gases diffusion inside the FC. The limitation of the methods described above is that they cannot reproduce some behaviors of the FC impedance spectrum, in particular with respect to the $45^{\circ}$ slope that appears around certain frequencies as noted in 20, 22 and 30. As mentioned before [19], to simulate reality much better, it is possible to add a large number of RC circuits, thus increasing the model order and therefore the number of parameters to identify as well as the model calculation time. Alternatively, recent works have suggested PEMFC modelling based on Fractional Order Models (FOM). This approach enables to reproduce more precisely the $45^{\circ}$ slope while being more compact than the previous models [30, 31, 21]. Following the analysis, the latter method is favored in this fractional order transfer function is obtained approximating the diffusion impedance by Taylor series. This technique permits us to implement an innovative identification method easy to execute in real time and thus well-suited to the final on-line diagnosis goal. Figure 1 gives a basic schematic of a PEMFC. It is fabricated by stacking bipolar plates, Gas Diffusion Layer (GDL), Active Layer (AL) and a polymer proton exchange membrane. On both sides, gases flows and diffuses through GDL and AL. the electrochemical reactions take place at the inter-phase between AL and membrane.

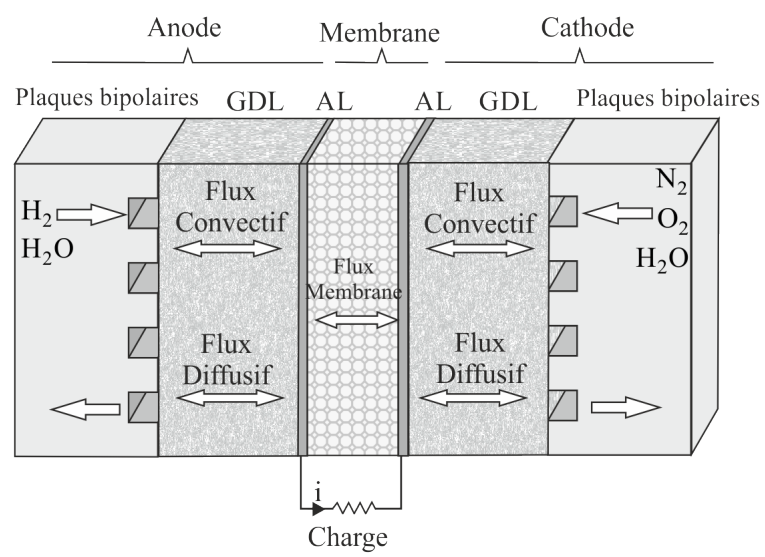

Figure 1: Fuel cell

From a user's perspective, a FC is modelled by the voltage at its terminals which is the sum of its theoretical voltage and the diffusion, activation and ohmic voltage drops.
This voltage is given by:

$$
V_{\text {cell }}=U_{t h}-\eta_{\text {act }}-\left|\eta_{\text {diff }}\right|-R_{m} I
$$

First, the theoretical potential of the $\mathrm{FC}$ is a function of $\mathrm{H}_{2} / \mathrm{O}_{2}$ Gibbs free energy which depends on the temperature and the partial pressures of oxygen in the cathode side and hydrogen in the anode side. It is given by the following relation:

$$
U_{t h}=U_{0}+\frac{R T}{n F} \log \left(P_{H_{2}}\left(P_{O_{2}}\right)^{0.5}\right)
$$

Second, the kinetics of the redox reaction causes activation phenomena occurring in the active layers (AL). These phenomena result in losses represented by the Tafel law:

$$
\eta_{a c t}=-\frac{R T}{\alpha n F} \log \left(\frac{I}{I_{0}}\right)
$$

The linearization of equation (3) around a steady state operating current leads to describe activation phenomena losses using a resistance given by:

$$
R_{t}=\frac{\partial\left|n_{a c t}\right|}{\partial I_{a c t}}
$$

Third, ohmic phenomena are mainly due to the proton conduction in the membrane, depending on the rate of hydration and temperature. The FC ohmic resistance is represented by a resistor

$$
R_{m}=\frac{l}{S \sigma\left(T, \lambda_{m}\right)}
$$

Where $\mathrm{S}$ is the membrane area and $\sigma$ its protonic conductivity. This latter strongly depends on temperature $\mathrm{T}$ and membrane hydration rate $\lambda_{m}$ as illustrated by the following relations 32

$$
\sigma=\left(0,005139 \lambda_{m}-0.00326\right) e^{1268\left(\frac{1}{303}-\frac{1}{T}\right)}
$$

The membrane hydration rate is given by 25 as the ratio between the number of water molecules and the number of sulphonic sites of the polymer:

$$
\lambda_{m}=\frac{n_{\mathrm{H}_{2} \mathrm{O}}}{n_{\mathrm{SO}_{3}^{-}}}
$$

Where $n_{\mathrm{H}_{2} \mathrm{O}}$ is the number of water molecules in the membrane and $n_{\mathrm{SO}_{3}^{-}}$is the number of sulphonic sites of the polymer. The gases flow through the channels of the bipolar plates by convection as depicted in fig.1. Once at the GDL, they diffuse to the AL and reach the reactive sites. This article assumes that the diffusion is uniform in the both layers, GDL and AL, and the consumption rate of gas is uniform over the entire active surface. The propagation direction is defined from the GDL to the membrane. Assuming that the species diffuses in a biphasic medium 
of liquid water and vapor and defining a diffusion coefficient to identify experimentally, most studies describe the diffusion using Fick's second law:

$$
\frac{\partial C_{i}(x, t)}{\partial t}=D_{i} \frac{\partial^{2} C_{i}(x, t)}{\partial x^{2}}
$$

Solving this diffusion equation in the Laplace domain defines an impedance of concentration-diffusion known as "Warburg impedance" [17] given by:

$$
Z_{W}=R_{d} \frac{\tanh (\sqrt{\tau s})}{(\sqrt{\tau s})}
$$

Based on equations 2, 3, 4, 8, eaquation 1 can be represented by Fig.2. Indeed, in this model, the activation phenomena losses and the membrane resistivity are described by two resistances Rt and Rm respectively, while the diffusion phenomena losses 8 are modeled by Warburg impedance $Z_{W}$. Finally the accumulation of protons and electrons at both cathode and anode membrane/AL interfaces is modelled by the so-called double layer capacitor.

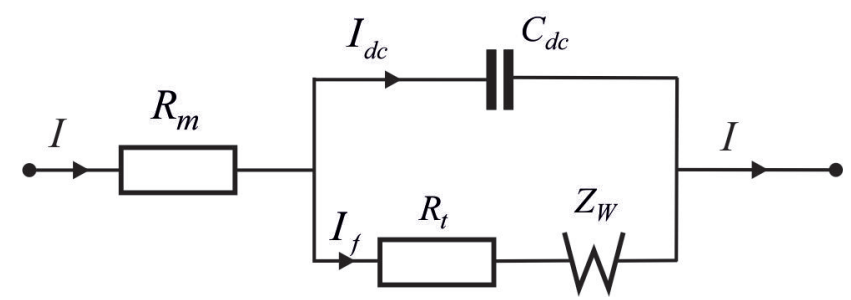

Figure 2: PEMFC equivalent electrical circuit model (Randles)

In order to obtain an explicit I-V transfer function, the latter model will be refined.

\section{PEMFC explicit fractional order impedance model}

With the aim of on-line monitoring, a compact and simple mathematical model has to be exhibited. In particular, the Warburg impedance requires a specific attention. As it is a function of a hyperbolic tangent of a non-integer or-230 der, it can be approximated using Taylor series to deduce an explicit transfer function model. Indeed, Iftikhar 30. and Sailler 31 proposed to approximate 33 the Warburg impedance by:

$$
Z_{W}=R \frac{\tanh (\sqrt{s \tau})}{\sqrt{s \tau}}=R \frac{\sinh (\sqrt{s \tau})}{\cosh (\sqrt{s \tau})} \cdot \frac{1}{\sqrt{s \tau}} \cong \frac{R}{\sqrt{1+s \tau}}
$$

The square root will be approximated in this new rela-240 tion by Taylor series to obtain distinct and explicit orders model. Considering the first three terms of the expansion, the root square in 10 can be written as :

$$
\sqrt{1+x} \cong \sqrt{x}+\frac{\sqrt{\frac{1}{x}}}{2}-\frac{1}{8}\left(\frac{1}{x}\right)^{\frac{3}{2}}+\mathcal{O}\left(x^{3}\right)
$$

Figure 3 shows the error between the precise expression of Warburg impedance 9 and its final approximation 10. It shows that it is very accurate except at very low frequency.

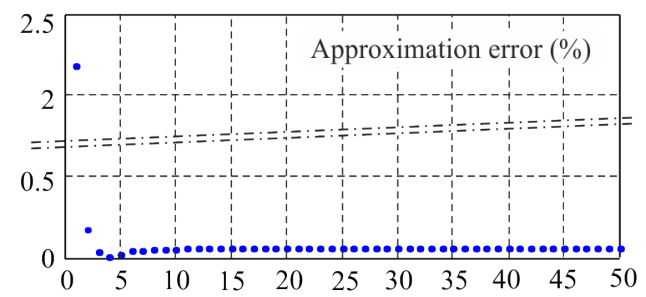

Figure 3: error of Warburg impedance approximation

From figure 2, the transfer function of the PEMFC impedance can be given by:

$$
H(s)=\frac{1}{\frac{1}{R_{t}+Z_{w}}+s C_{d c}}+R_{m e m}
$$

Consequently, based on 10 and 11, the suggested FC transfer function exhibits explicit orders:

$$
H(s)=\frac{b_{0}+b_{1} s+b_{2} s^{\frac{3}{2}}+b_{3} s^{2}+b_{4} s^{\frac{5}{2}}+b_{5} s^{3}}{1+a_{1} s+a_{2} s^{2}+a_{3} s^{\frac{5}{2}}+a_{4} s^{3}}
$$

$\mathrm{H}(\mathrm{s})$ is characterized by only ten coefficients $a_{i}$ and $b_{j}$ which are obviously function of the physical parameters.

$$
\begin{aligned}
& a_{i}=f_{i}\left(R, R_{t}, R_{m e m}, C_{D C}, \tau\right) ; i=0, \ldots, 4 \\
& b_{j}=g_{j}\left(R, R_{t}, R_{m e m}, C_{D C}, \tau\right) ; j=0, \ldots, 5
\end{aligned}
$$

In sum, this first part permitted to develop a PEMFC model closely based on the system structure and its physical phenomena. Hence, it can assist in understanding the FC evolutions. More specifically, as shown in tab.1, the models parameters change regarding water flooded or dry operating conditions. For these very important PEMFC defaults, the most relevant parameters are a3, b2 and b4. Indeed, these three parameters increase significantly in the They can be considered appropriate indicators of the critical track defects. That is why the study objective is to develop identification methods able to estimate the model's parameters and diagnose the operating state of the FC, referring to the parameters evolution. However for this purpose and greater certainty, experimental data are needed. That is why a test bench was developed and used. 


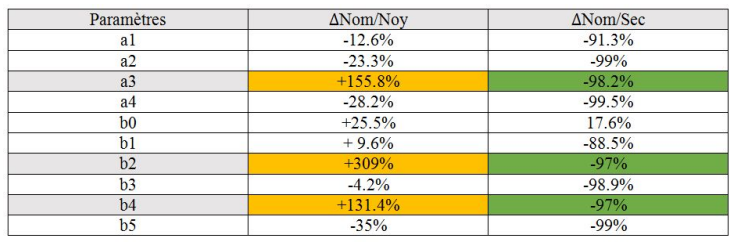

Figure 4: Explicit fractional order parameters evolution in dry and water flooded operating conditions

\section{Experimental setup}

Experimental measurements of the impedance spectrum are performed on a $100 \mathrm{~cm} 2$ active area single FC (ZSW, Germany) working with an open anode. It is fed with pure hydrogen at the anode inlet while humidified ambient air is supplied at the cathode side. The test bench is managed by a supervision algorithm implemented in a dSpace control board. Among other things, it allows control of it permits to control the $\mathrm{FC}$ humidity rate by both ${ }^{27}$ monitoring FC cooling temperature (TFC) and humidifier (TAH). The chemical energy transformed by the cell into electrical energy is absorbed by an active load (ZS1806 made by Hoecherland Hackl). Figure 4 and 5 shows a picture and a block diagram of the test bench developed at the GeePs. Finally the electronic load can be monitored by Fuel Cell Impedance Analyzer FC350 designed by the Gamry Company. It allows to impose a static steady current and superimpose a sinusoidal current signal from 10 $m \mathrm{~Hz}$ to $300 \mathrm{kHz}$. It also enables electric (VFC, IFC) data acquisition and processes these signals to calculate the $\mathrm{FC}$ impedance. This latter technique is called the Electrochemical Impedance Spectroscopy (EIS). EIS is performed over a frequency range of $0.1 \mathrm{~Hz}$ to $1 \mathrm{kHz}$ around several operating points: from $5 A$ to $40 A$ with $5 A$ increments. Indeed, the FC impedance spectrum changes with the considered operating point.

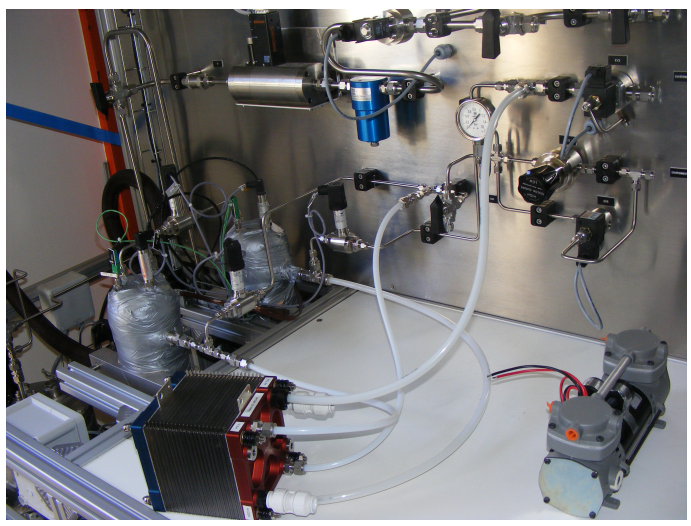

Figure 5: Test bench picture at GeePs

For instance, figure 7 shows the Nyquist diagram of 270 the PEMFC impedance spectrum for an EIS conducted

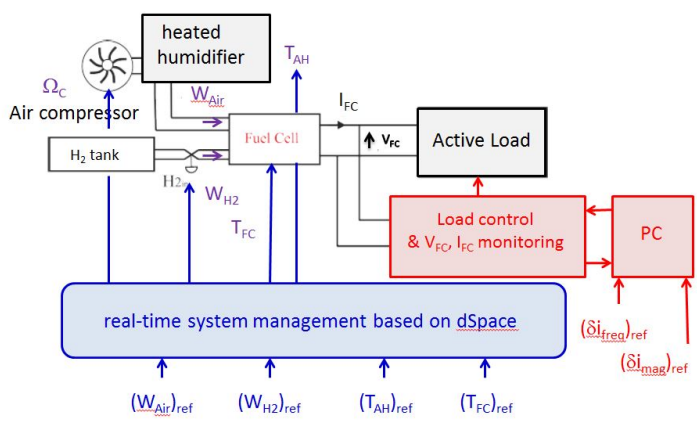

Figure 6: Block diagram of the test bench

around a polarization current of $15 \mathrm{~A}$. On this plot, the FC impedance moves from the right side to the left side as far as the frequency increases. At high frequency, a $45^{\circ}$ slope can be noticed as a typical pattern of diffusion mechanisms

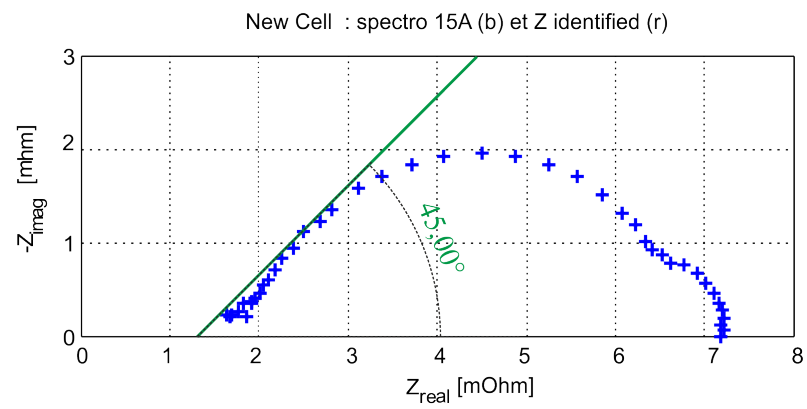

Figure 7: : PEMFC experimental impedance spectroscopy $I 0=15 \mathrm{~A}$

\section{Fractional order model validation}

Before developing specific methods, our first attempt is to assess if the suggested compact model is representing the FC accurately. To do so it is necessary to obtain its ten parameters from data. In this section, the PEMFC FOM is identified using a frequential identification method based on genetic algorithms: This method identify the model from the PEMFC impedance spectrum obtained using EIS measurements. A first identification by genetic algorithm is initiated to determine a suitable starting point for the optimization algorithm. Which avoid the nonlinear optimization from starting the optimization randomly. The frequential methods aims here to validate the proposed fractional order model.

\subsection{Frequential identification method}

This section discusses the parametric identification using a multidimensional unconstrained nonlinear minimization. This approach is based on the minimization of 
a quadratic criterion which represents the difference be-

$$
J=0.5\left(\sum\left(\Re_{Z_{\text {exp }}}-\Re_{Z_{\text {ident }}}\right)^{2}+\sum\left(\Im_{Z_{\text {exp }}}-\Im_{Z_{\text {ident }}}\right)^{2}\right)^{\frac{1}{2}}
$$

This method is sensitive to its initial conditions. For this ${ }^{325}$ purpose, Phlippoteau 22 proposes to initialize the identification algorithm using known parameters values given in the literature or deduced from the impedance spectrum geometry in the Nyquist plane. To go even farther in this approach, the suggested identification method relies on $\mathrm{a}^{330}$ two-step process: using a genetic based algorithm first and second operating the nonlinear optimization initialized with the first result. In this way nonlinear optimization algorithm, which will refine the genetics algorithm results while the genetic algorithm is used to prevent the optimization algorithm to stop in a local minimum point. Finally, the identification algorithm procedure is presented

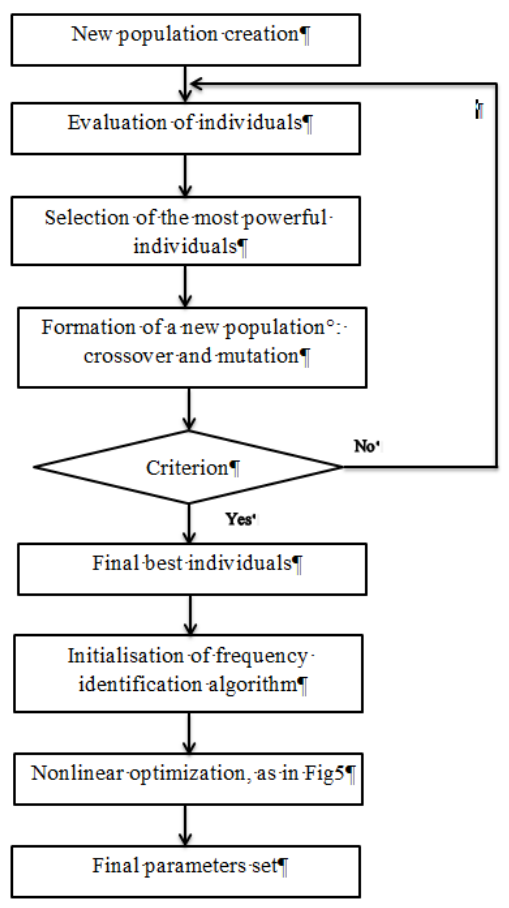

Figure 8: : Genetic algorithm and nonlinear optimization flowchart

\subsection{Identification results}

This method has been tested using impedance spectroscopy data made on a single FC using the test bench described previously. Figure 9 presents the identifica-

tion results using only the genetic algorithms. Figure 10,40 presents the identification result using only the nonlinear optimization initialized with zero. Finally, figures 11 and
12 present the identification results using the optimization method described in this paper (genetic algorithm 320 we nonlinear optimization technique) All the figures compare the identified impedance model's spectrum (red) to the experimental one (blue). The identification results using the suggested method are much more accurate than those based either on genetic algorithm or nonlinear optimization routine. Last but not least, the fractional order model presents the advantage to be compact: it describes accurately the $\mathrm{FC}$ behavior using a small number of parameters. Moreover it represents exactly the $45^{\circ}$ slop which appears at high frequencies on the impedance diagram plotted in Nyquist plan and succeeds in following the real $\mathrm{FC}$ at low frequency.

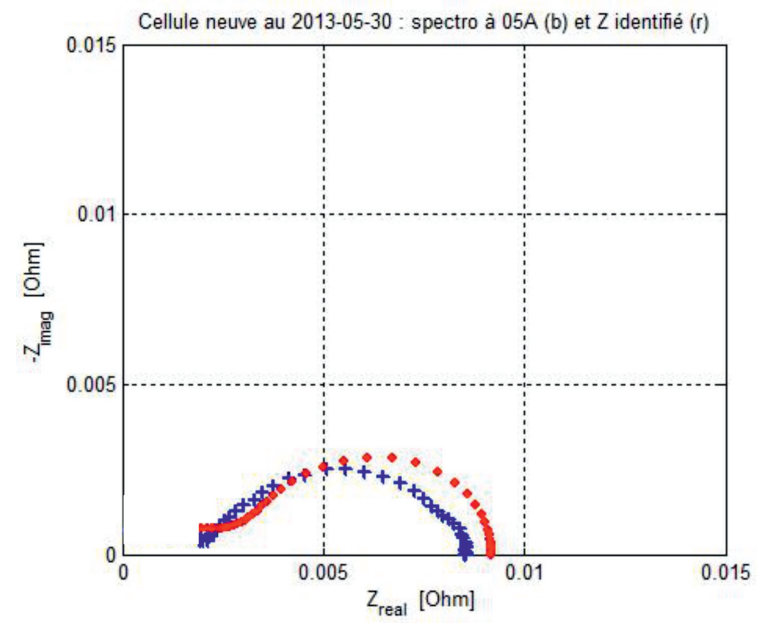

Figure 9: : Genetic algorithm results, for an impedance spectroscopy measured at $10 \mathrm{~A}$

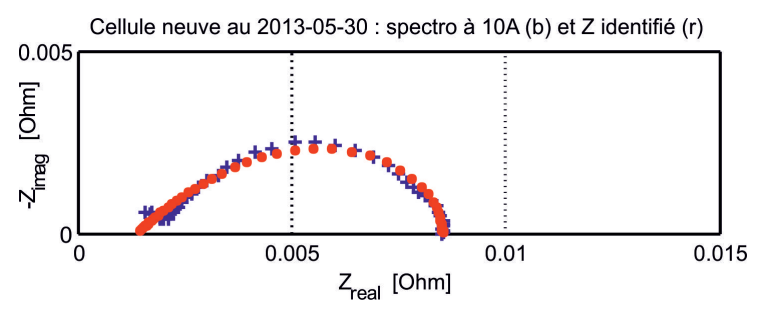

Figure 10: : Nonlinear Optimization results initialized with zero, for an impedance spectroscopy measured at $10 \mathrm{~A}$

Figure 11 and 12 show the impedance spectrum shape change with the operating point. (10A and 35A) Evidently. Indeed, at middle and low frequencies, the volume of second half circle increases. This is due to the activation and diffusion phenomena.

In a nutshell, section $\mathrm{V}$ shows that the FOM seems to be well adapted to describe the FC behavior. Furthermore, it is characterized by a low number of parameters. This proposal offers a good compromise between accuracy and compactness. To address the FC diagnosis purpose, the issue is to find a viable solution able to extract in short 
time the aforementioned parameters. It is contemplated in the next section.

\section{Time series parameters identification}

New Cell : spectro $10 \mathrm{~A}(\mathrm{~b})$ et $Z$ identified $(r)$

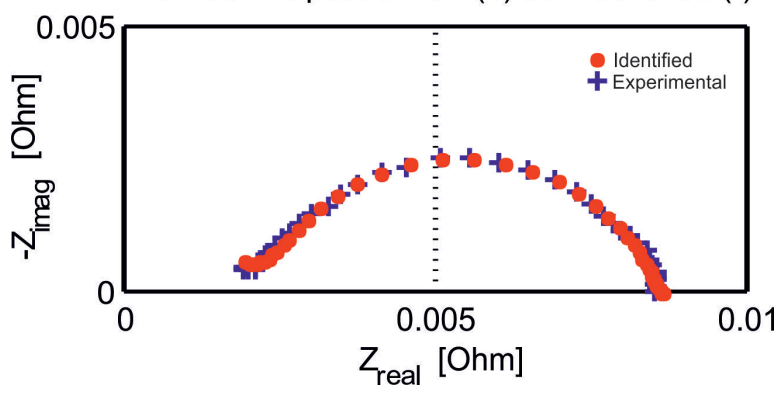

Figure 11: : Frequential identification results using impedance spectroscopy data measured at $10 \mathrm{~A}$

Experimental

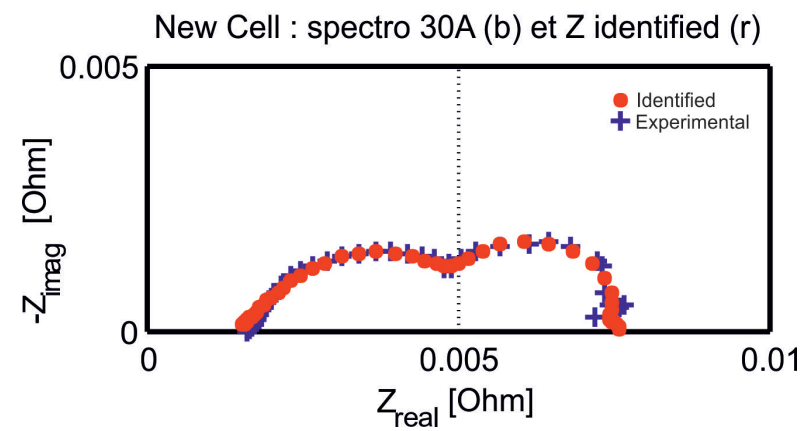

Figure 12: : Identification results using impedance spectroscopy data measured at $35 \mathrm{~A}$
A diagnosis method can be built by monitoring the evolution of the model parameters. Indeed, the parameters values change with the operating conditions. Using experimental data, the model parameters can be identified and compared to their reference values, in nominal, dry and flooding cases. For online diagnosis in embedded applications, electrochemical impedance spectroscopy (EIS) is not well adapted to characterize the fuel cell. For online FC state monitoring, EIS is not well adapted. Indeed, it results in a slow process scanning harmonic signals on a wide frequency range and moreover relates on a steady state assumption which is clearly unrealistic. Nevertheless, Alternatively temporal data series can be used to identify the model parameters. The vehicle load and. manded power may present a sufficiently high spectral content which can be enough allows continuously updating extract FC fractional model parameters convenient using current/voltage data for this identification. Conversely, in steady state mode, a small amplitude PRBS current of small amplitude can be superimposed to excite the system and extract dynamic voltage response. This method will be tested in this section.

In this section, the FOM will be reformulated to be adapted to classical identification methods as the least square method. The present section is broken down in two parts. For offline diagnosis purpose, the model parameters will be first identified using a least square method. Later,Then, for online diagnosis application, a recursive least square method adapted to FOM will is derived to identify the parameters.

\subsection{Fractional order least square method for FOM offline diagnosis purposes}

For offline diagnosis, least square method can be used to find the parameters values. Offline diagnosis can be done post-use, in maintenance or retarded, after saving enough data needed to identify the parameters. Here, a least square method adapted to FOM is used to identify the model parameters [34, 35, 36, 37, 38: This method uses time-series current and voltage. A PRBS is applied at a polarization PEMFC current point. The PEMFC voltage response is then measured and used to identify the model's parameters.

A fractional order model can be described by the equa-

$$
\sum_{l=0}^{L} a_{l} D^{n_{a_{1}}} y(t)=\sum_{m=0}^{M} b_{m} D^{n_{b_{m}}} u(t)
$$

With $a_{0}=1$ 
$n_{a_{l}}, n_{b_{m}}$ : are real positive numbers, integers or nointegers (fractions) and supposed known. $a_{l}, b_{m}$ : are the 410 coefficients of the derivative operators supposed unknown.

The discretization of a Fractional Order Derivative (FOD) is done using Grunwald's [37, 36] approximation

$$
[D x(t)]^{n}=\frac{1}{h^{n}} \sum_{k=0}^{K}(-1)^{k}\left(\begin{array}{c}
n \\
k
\end{array}\right) x[K-k]
$$

The discretized model obtained with Grunwald formula can be written as in $\mathrm{Eq} 18$, where the output sample $y[K]$ is a function of the previous inputs and outputs.

$$
\begin{gathered}
y[K]=-\frac{\sum_{l=0}^{L} \frac{a_{l}}{h^{n_{a}} a_{l}}\left[\sum_{k=1}^{K}(-1)^{k} C_{n_{a_{l}}}^{k} y[K-k]\right]}{\sum_{l=0}^{L} \frac{a_{l}}{h^{n} a_{l}}}+ \\
\frac{\sum_{m=0}^{M} \frac{b_{m}}{h^{n} b m} \sum_{k=0}^{K}(-1)^{k} C_{n_{b}}^{k} u[K-k]}{\sum_{l=0}^{L} \frac{a_{l}}{h^{n} a_{l}}}
\end{gathered}
$$

This relation presents a non-linearity with respect to parameters. To deduce a linear form, a variable change is used to define a new parameters set $\left(a_{0}^{\prime}, \ldots, a_{L}^{\prime}, b_{0}^{\prime}, \ldots, b_{M}^{\prime}\right)$ where 36]:

$$
a_{l}^{\prime}=\frac{\frac{a_{l}}{h^{n} a_{l}}}{\sum_{i=0}^{L} \frac{a_{i}}{h^{n a_{i}}}} \quad ; \quad b_{m}^{\prime}=\frac{\frac{b_{m}}{h^{n} b_{m}}}{\sum_{i=0}^{L} \frac{a_{i}}{h^{n} a_{i}}}
$$

The new model expression is then:

$$
y[K]=-\sum_{l=0}^{L} a_{l}^{\prime} Y_{l}[K]+\sum_{m=0}^{M} b_{m}^{\prime} U_{m}[K]
$$

With,

$Y_{l}[K]=\sum_{k=1}^{K}(-1)^{k} C_{n_{a_{l}}}^{k} y[K-k], \quad U_{m}[K]=\sum_{k=0}^{K}(-1)^{k} C_{n_{b_{m}}}^{k} u[$

$$
0 \leq l \leq L, 0 \leq m \leq M
$$

Equation 21 is developed as :

$$
\hat{y}\left[K, \hat{\theta}_{r}\right]=-\hat{a}_{0}^{\prime} Y_{0}[K]-\sum_{l=1}^{L} \hat{a}_{l}^{\prime} Y_{l}[K]+\sum_{m=0}^{M} \hat{b}_{m}^{\prime} U_{m}[K]
$$

Using the constraint $\sum_{l=0}^{L} a_{l}^{\prime}=1$ permits to eliminate $a_{0}^{\prime}$

$\hat{y}\left[K, \hat{\theta}_{r}\right]=-\left(1-\sum_{l=1}^{L} \hat{a}_{l}^{\prime}\right) Y_{0}[K]-\sum_{l=1}^{L} \hat{a}_{l}^{\prime} Y_{l}[K]+\sum_{m=0}^{M} \hat{b}_{m}^{\prime} U_{m}\left[\Psi^{\circ}\right]$
The model output is hence linearly expressed with respect to the vector of new parameters:

$\hat{y}\left[K, \hat{\theta}_{r}\right]=-Y_{0}[K]-\sum_{l=1}^{L} \hat{a}_{l}^{\prime}\left(Y_{l}[K]-Y_{0}[K]\right)+\sum_{m=0}^{M} \hat{b}_{m}^{\prime} U_{m}[K]$

For $N$ measurement points between $K h$ and $(K+N) h$ linear matrix equation is given by:

$$
\hat{Y}\left(\hat{\theta}_{r}\right)=-Y_{0}+\varphi \hat{\theta}_{r}
$$

Where $\phi$ is composed by $-Y_{i}[K]+Y_{0}[K]$ to $-Y_{1}[K+$ $N]+Y_{0}[K+N], i=1 \ldots L$ and $U_{j}[K]$ to $U_{j}[K+N]$

The vector of parameters is given by:

$$
\hat{\theta}_{r_{\text {opt }}}=\left(\varphi^{T} \varphi\right)^{-1} \varphi^{T}\left(Y+Y_{0}\right)
$$

Finally initial parameters are computed by reversing the previous variable change using the following relations [37, [36] :

$A=\left[\begin{array}{ccc}\left(a_{1}^{\prime}-1\right) h^{-n_{a_{1}}} & \cdots & a_{1}^{\prime} h^{-n_{a L}} \\ a_{2}^{\prime} h^{-n_{a 1}} & \cdots & a_{2}^{\prime} h^{-n_{a_{L}}} \\ \vdots & \vdots & \vdots \\ a_{L}^{\prime} h^{-n_{a 1}} & \cdots & \left(a^{\prime}{ }_{L}-1\right) h^{-n_{a_{L}}}\end{array}\right] \Rightarrow A *\left[\begin{array}{c}a_{1} \\ a_{2} \\ \vdots \\ a_{L}\end{array}\right]=$

$$
b_{m}=b_{m}^{\prime} \sum_{l=0}^{L} a_{l} h^{n_{b_{m}}-n_{a_{l}}}
$$

This algorithm was implemented to identify the PEMFC FOM parameters. The identified model simulation results are presented in the next section.

\section{Offline identification results}

The identification data had been obtained on a signal [Kelt vising using the PEMFC response to a PRBS current excitation of $1 \mathrm{~A}$ around a $7 \mathrm{~A}$ polarization current. The identification results using the previous method experimental results are presented in figure ??. The identified model reproduces correctly the FC voltage response.

To test the parameters values, identification data had (The PEMFC response to a PRBS current excitation of $1 \mathrm{~A}$ around a $7 \mathrm{~A}$ polarization current had been simulated . From the parameter evolution given by 4 in the dried and flooded cas, three models had been simulated to generate identification data in these cases. The data had been used later to identify the parameters in the three cases (nominal, dry, flooded).

Tab 1 present the comparison between the identified parameters and the model's parameters (used to simulate the identification signal), in the dry, flooded and nominal cases. As an example, the parameters $a_{3}$ are identified correctly. 


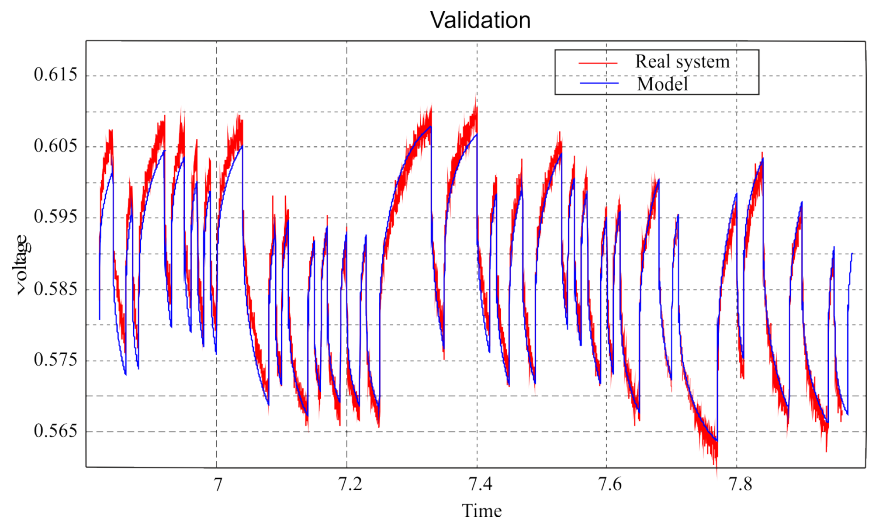

Figure 13: : Identification results using least square method for FOM and experimental data measured at $7 \mathrm{~A}$

With regards to the identification results, it can be concluded that the time-series identification results are a first step to off-line diagnosis and reinforce the idea that the recursive least square method adapted to fractional order models can be applied for on line diagnosis using the previous model.

With this method, the parameters can be identified off line (post-operating) using the experimental data saved during the operating period. The identified parameters can be used to establish an offline diagnosis by comparing their identified values to their references values (in a nominal, dried and drown operating cases)

\section{9. on line identification for diagnosis purpose :pur- ${ }^{48}$ pose: MCR}

An on line diagnosis method can be build based on recursive identification methods, which identifies the model parameters online. Recursive least square (RLS) method can be used [39] to identify the model parameters using the model reformulation given previously by the equations 16 to 24.

The equation 24 has the following linear form:

$$
y((k+1) h)=\theta^{t} \phi(k)
$$

$\theta$ is the vector of parameters to be identified and $\phi(k)$ the measurement matrix, such that:

$$
\phi(k)=\left[-Y_{1}(k), \ldots,-Y_{5}(k), U_{0}(k), \ldots, U_{6}(k)\right]
$$

Table 1: Comparison between the identified parameters and the model's parameters

\begin{tabular}{llll}
$a_{3}$ & Nominal & flooded & dry \\
\hline ModelParameter & $?$ & $?$ & $?$ \\
\hline IdentifiedParameter & $?$ & $?$ & $?$ \\
\hline
\end{tabular}

The prediction equation is

$$
\hat{y}(k+1)=\hat{\theta}_{k+1}^{t} \phi(k)
$$

The parameter vector is estimated by minimizing a quadratic criterion:

$$
\hat{\theta}_{k}=\arg \min _{\theta} \frac{1}{k} \sum_{k=1}^{K}[y(k)-\hat{y}(k, \theta)]^{2}
$$

The solution to this problem [39] is given by:

$$
\hat{\theta}(K)=\left[\sum_{k=1}^{K} \phi(k) \phi^{T}(k)\right]^{-1} \sum_{k=1}^{K} \phi(k)\left[y(k)-Y_{0}(k)\right]
$$

The algorithm of the recursive least square method is then implanted using the following relations at each iteration:

$$
\begin{aligned}
& \hat{\theta}_{k}=\hat{\theta}_{k-1}+\frac{F(k-1) \phi(k)\left(y(k)-\left[\hat{\theta}_{k-1}^{T} \phi(k)-Y_{0}(k)\right]\right)}{1+\phi^{T}(k) F(k-1) \phi(k)} \\
& F(k)=F(k-1)-\frac{F(k-1) \phi(k) \phi^{T}(k) F(k-1)}{1+\phi^{T}(k) F(k-1) \phi(k)}
\end{aligned}
$$

The data used to identify the FOM, with the previous method, are current/voltage temporal series measurements. The control signal is a Pseudo-Random Binary Sequence (PRBS) with an amplitude $\delta I=1(A)$, applied around a current of polarization $\mathrm{I}_{0}=7[A]$.

The parameters identification using this method and time series experimental data permit to mimic correctly the experimental output voltage for slow and quick variations, and enable reproducing correctly the shape of the impedance spectrum, as shown in fig 14 and 15 , respectively.

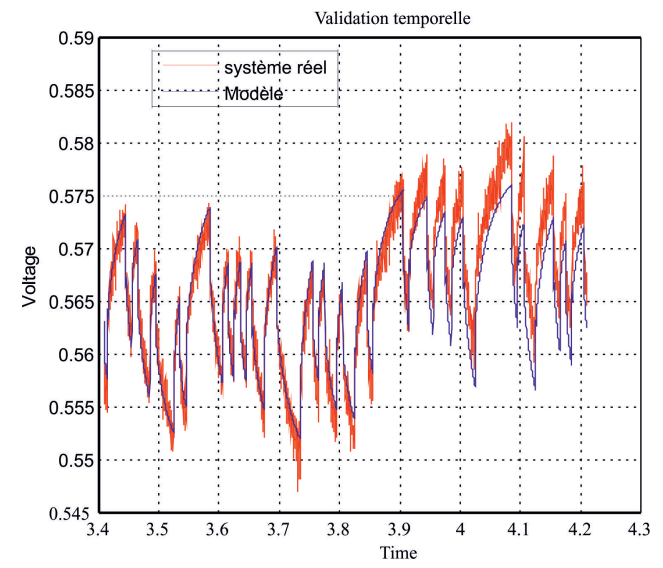

Figure 14: : online identification validation of the FOM using RLS method for FOM

The parameters identified using this method are composed by the physical parameters of the PEMFC. The solution of equations to go back to the FC physical parameters (Rd, Cdc, Rt..etc) is not unique. It is therefore not 


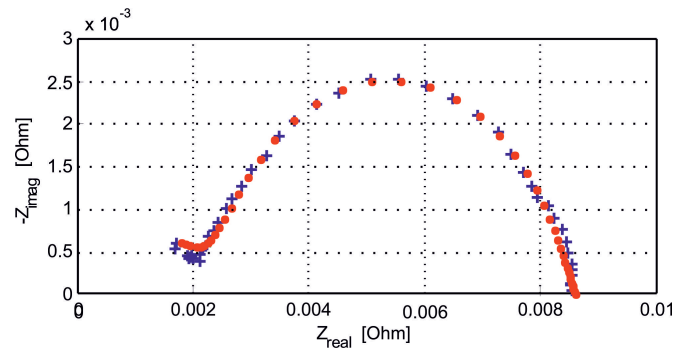

530

Figure 15: : online identification frequential validation of the FOM using RLS method for FOM

possible, to find the physical parameters using the parameters identified by this method, A diagnosis by monitoring the evolution of the physical parameters reflecting certain ${ }^{545}$ phenomena is not possible.

It is nevertheless possible to achieve a sensitivity analysis of the transfer function parameters to the changes of operating conditions. It is then possible to perform a diag-550 nosis based on the values of the identified transfer function parameters, considering as reference their values in a nominal, dry and flooding cases.

\section{Conclusion}

This paper presented the modeling and identification of a PEMFC fractional order model in the perspective of the ${ }^{560}$ PEMFC state monitoring. It presented a new explicit fractional order transfer function model. To identify directly the impedance spectrum form and validate the presented model, a frequency identification method had been used ${ }^{565}$ to find the FOM parameters which allow reproducing the impedance form. The nonlinear optimization key point is the initialization of the identification algorithm. The paper discusses then an initialization strategy which al- ${ }^{570}$ lows finding better results. The frequency identification results had been compared with experimental impedance spectrum which leads to the conclusion that the fractional order model is well adapted to describe a $\mathrm{PEMFC}^{575}$ impedance. For on-line diagnosis method, only time-series measurements can be technically used. The second part of this paper presented an identification method based on time series data. A least square method, adapted to frac- ${ }^{58}$ tional order models, had been implemented. The obtained results shown a good consistency with PEMFC simulated voltage response. This method does not use directly the FC experimental impedance spectrum data, but is tech- ${ }^{585}$ nically feasible on a FC placed in a car for an on-line diagnosis goal, unlike an EIS. Time-series identification by least square method adapted to fractional order gives good results which suggests that for future on line monitoring, recursive least square method is well suited for fractional order systems. As on-line monitoring methods can only rely on time-series measurements, these first pieces of evidence are key-points for the ongoing work. They indeed confirm that the FOM mimics well the FC behavior and with the objective of on-line diagnosis, recursive least square adapted to FOM can be an efficient tool to identify the model's parameters.

\section{References}

[1] Jing S. Rui LSXDLQZ. Local resolved investigation of pemfc performance degradation mechanism during dynamic driving cycle,. International Journal of Hydrogen Energy xxx 1 -12 2016 ;.

[2] Yousfi Steiner N. Hissel DMPCD. Diagnosis of polymer electrolyte fuel cells failure modes (flooding \& drying out) by neural networks modeling. International Journal of Hydrogen Energy 36306730752011 ;

[3] Bozorgnezhad A. Shams MKHHMAG. The experimental study of water management in the cathode channel of singleserpentine transparent proton exchange membrane fuel cell by direct visualization. International journal of hydrogen energy 40 2808-2832 2015;

[4] Fukuhara S. Marx NEKBLAAYBM. A lumped fluidic model of an anode chamber for fault tolerant strategy design. International Journal of Hydrogen Energy xxx 1-11 2016;.

[5] Kim H. Kim K. Numerical study on the effects of gas humidity on proton-exchange membrane fuel cell performance. International Journal of Hydrogen Energy xxx 1-8 2016;

[6] Shimpalee S. Van Zee J. Numerical studies on rib \& channel dimension of flow-field on pemfc performance. International Journal of Hydrogen Energy 32842856 2007;

[7] Giurgea S. Tirnovan RHDOR. An analysis of fluidic voltage statistical correlation for a diagnosis of pem fuel cell flooding. International Journal of Hydrogen Energy 38 4689-4696 2013;.

[8] Owejana J PTTAJDLAMKSG. Effects of flowfield and diffusion layer properties on water accumulation in a pem fuel cell. International Journal of Hydrogen Energy 3244894502 2007;

[9] Yana X. Houb MSLLBSQXHMPYB. Ac impedance characteristics of a $2 \mathrm{kwpem}$ fuel cell stack under different operating conditions and load changes. International Journal of Hydrogen Energy 3243584364 2007;

[10] Jouin M. Gouriveau RHDPMC, Zerhouni N. Phm of protonexchange membrane fuel cells - a review,. Chemical Engineering Transactions 331009 2013;

[11] Fouquet N. Doulet C. NCDTG, B OB. Model based pem fuel cell state-of-health monitoring via ac impedance measurements. Journal of Power Sources 159905913 (2006);

[12] Petrone R. Zheng ZHDPMCPCSMBMYSM. A review on model-based diagnosis methodologies for pemfcs. International Journal of Hydrogen Energy 38 7077-7091 2013;.

[13] Zheng Z. Petrone RPMCHDBMPCYSNSM. A review on nonmodel based diagnosis methodologies for pem fuel cell stacks and systems. International Journal of Hydrogen Energy 38 89148926 2013;.

[14] Wua I. Yuana XWHBMJJMZJ. Diagnostic tools in pem fuel cell research: Part ii physical/chemical methods. International Journal of Hydrogen Energy 3317471757 2008;.

[15] B?y?koglu A. Review of proton exchange membrane fuel cell models,. International Journal of Hydrogen Energy 301181 12122005 ;.

[16] Fontes G. TCSRMTAA. Interactions between fuel cell and power converters influence of current harmonics on a fuel cell stack. Annual IEEE Power Electronics Specialists Conference, 53, 4729-4735 2004;.

[17] Fontes G. Modelisation et caracterisation de la pile pem pour letude des interactions avec les convertisseurs statiques. Ph.D. thesis; The National Polytechnic Institute of Toulouse, France,; 2005.

[18] Sadli I TPMJPRSDB. Behaviour of a pemfc supplying a low voltage static converter,. Journal of Power Sources, Vol 156, no 1, pp 119-125 19 May 2006,;.

[19] Sadli I UMHMMJRSDB. Contributions of fractional differentiation to the modeling of electric double layer capacitance. En- 
ergy Conversion and Management, Volume 51, Issue 12, pages 2993-2999 December 2012;.

[20] Sadli. I P. Thounthong JPMSRBD. Behaviour of a pemfc supplying a low voltage static converter,. Journal of Power Sources, Volume 156, Issue 1, Pages 119-125 19 May 2006;.

[21] Cao H. Deng ZLXYJQY. Dynamic modeling of electrical characteristics of solid oxide fuel cells using fractional derivatives,. International Journal of Hydrogen Energy 3517491758 2010;.

[22] Phlippoteau V. Outils et methodes pour le diagnostic dun etat de sante dune pile a combustible. Ph.D. thesis; University of Toulouse (Institut National Polytechnique de Toulouse); 2009.

[23] Asghari S. MASM. Study of pem fuel cell performance by electrochemical impedance spectroscopy,. international journal of hydrogen energy 35 9283-9290 2010;.

[24] Kurz T. Alex Hakenjos JKMZCA. An impedance-based predictive control strategy for the state-of-health of pem fuel cell stacks. ournal of Power Sources, Volume 180, Issue 2, Pages 742-747 1 June 2008,;

[25] Barbir F. PEM Fuel Cells - Theory and Practice, 2nd Edition. Academic Press; 09 Nov 2012.

[26] Mathias Gerard Jean-Philippe Poirot-Crouvezier DHMCP. Oxygen starvation analysis during air feeding faults in pemfc. International Journal of Hydrogen Energy, Volume 35, Issue 22, Pages 12295-12307 November 2010,;

[27] Turpin C Van Laethem D MBRORXVOCV. Modelling and analysis of an original direct hybridization of fuel cells and ultracapacitors. Mathematics and Computers in Simulation, In Press, Corrected Proof Available online 23 October 2015;.

[28] Hinaje M. Rael S. NPND, B. D. An equivalent electrical circuit model of proton exchange membrane fuel cells based on mathematical modelling. Energies, 5, 2724-2744 2012;.

[29] Noiying P Hinaje M TPRS, B. D. Using electrical analogy to describe mass and charge transport in pem fuel cell. Renewable Energy, 44, 128-140 2012;;

[30] Iftikhar M.U. RDDFRSBY, Retiere N. Dynamic modeling of proton exchange membrane fuel cell using non-integer derivatives. Journal of power sources, 160, 1170-1182 2006.;

[31] Sailler S. IMRDDFRSBY, Retiere N. Modelisation fractionnaire des piles a combustible de type pemf. 18eme Congre Franais de Mecanique, Grenoble, 27-31 Aout. 2007.;.

[32] Springer T. E. ZTA, Gottesfeld S. Polymer electrolyte fuel cell model. Jelectrochemsoc, vol-138, N8, August 1991;.

[33] Szymon Racewicz Delphine M. Riu NMRPJC. Half-order modeling of saturated synchronous machine,. IEEE Transactions on Industrial Electronics, Vol 61, no 10, pp 5241 - 5248, Oct. $2014, ;$

[34] Oustaloup A. Systemes asservis lineaires dordre fractionnaire : theorie et pratique. Editions MASSON.; 1983.

[35] Malti R. AM, Oustaloup A. Developpements mathematiques autour des modeles fractionnaires application l'identification de systemes. GT identification 2003.;.

[36] Victor S. Identification par modle non entier pour la poursuite robuste de trajectoire par platitude. Ph.D. thesis; (PhD thesis), University of Bordeaux 1.; 2010.

[37] Cois O. Systemes lineaires non entiers et identification par modele non entier : application en thermique, (phd thesis),. Ph.D. thesis; university Bordeaux 1.; 2002.

655 [38] Victor S. MRGOA. Parameter and differentiation order estimation in fractional models,. Automatica 49, 926935 2013;

[39] Abdelbaki Djouambi Alina Voda AC. Recursive prediction error identification of fractional order models,. communication in nonlinear science and numerical simulation, volume 17,251725242012, 